\title{
Perceptual-motor coordination and prism adaptation during locomotion: A control for head posture contributions
}

\author{
GORDON M. REDDING \\ Illinois State University, Normal, Illinois \\ and \\ BENJAMIN WALLACE \\ Cleveland State University, Cleveland, Ohio
}

\begin{abstract}
Adaptation to prismatic displacement was examined under conditions of locomotion in hallways. The relative magnitude of visual and proprioceptive shift depended upon the availability of visible sound sources in the hall environment. When the speaking experimenter was visible to the subject, visual shift was greater than proprioceptive shift, but when the experimenter was not visible, proprioceptive shift was greater than visual shift. The fact that this result was obtained for both leftward and rightward displacements, with which different head postures are expected, argues against an interpretation in terms of changing head posture and associated muscle potentiation of eye and neck. The results are consistent with a model that assumes that the locus of adaptive recalibration depends upon the direction of coordinative linkages between components of the total perceptual-motor system. Registration of the degree of discordance and adaptation are assumed to reside in the guided system(s) and the directionality of linkage is determined by task structure. Thus, visible sound sources provide for auditorially guided visual responses with consequent discordance registration and adaptation in the visual system. In the absence of visible sound sources, coordinative linkage of subsystems is assumed to consist in visual anticipation of proprioceptive encounters (e.g., first seeing a wall and then brushing it with the shoulder or arm) and consequent discordance registration and adaptation in the proprioceptive system.
\end{abstract}

The organization of the perceptual-motor system as it is used in many everyday situations may be conceptualized as depicted in Figure 1 (Redding, Clark, \& Wallace, 1985; Redding \& Wallace, 1985a, 1985b); this is the case, for example, when a person walks a familiar path, such as a hallway, while at the same time engaging in other activities, such as looking for a room number, at a speaking companion, or toward an obstacle just bumped into. Locomotion per se is assumed to be automatically ${ }^{1}$ guided via movement channels by Gibson-like optical flow patterns (e.g., Fitch, Tuller, \& Turvey, 1982; Lee \& Thomson, 1982; Longuet-Higgins \& Prazdny, 1980) and does not normally require central processing capacity (i.e., attention) unless nonroutine terrain is encountered. The continuous nature of optical flow is such that accurate locomotion can be maintained with occasional intentional visual response to auditory (ear-head) and/or proprioceptive (hand-head) stimuli (cf. Thomson, 1983). That is, a person can occasionally direct his/her eyes to look at,

\footnotetext{
The research was supported in part by Grant 1-R03-MH34383 from the National Institute for Mental Health to the second author. Correspondence should be addressed to Gordon M. Redding, Department of Psychology, Illinois State University, Normal, IL 61761.
}

for example, another person speaking (i.e., the ear-toeye linkage) or a just-touched obstacle (i.e., the hand-toeye linkage). In these examples, position information about the heard person or the touched obstacle is sent to the visual (eye-head) system, but of course, the direction of these guidance linkages can be strategically reversed in response to situational demands. For example, the person can reach for (hand-head system) a seen door (eyehead system) or turn the head (ear-head system) to better hear a seen speaker. Thus, perceptual-motor organization is strategically flexible, linking various subsystems in response to task demands. Some linkages, such as that required for walking, are so routine as to be effortless and can be performed simultaneously with other behavior. Nonroutine linkages, such as that involved in looking for an unexpected obstacle or speaker, require attention, and mutual interference occurs when more than one (or at most a very few) such behavior is attempted.

Prism adaptation, especially the variable nature of such adaptation, can be understood in the context of this general model of perceptual-motor organization. When a person walks about hallways while wearing prisms that laterally displace the visual field, locomotion is affected very little, because optical flow suffers little or no distortion by the prisms and walking can be guided in the normal auto- 


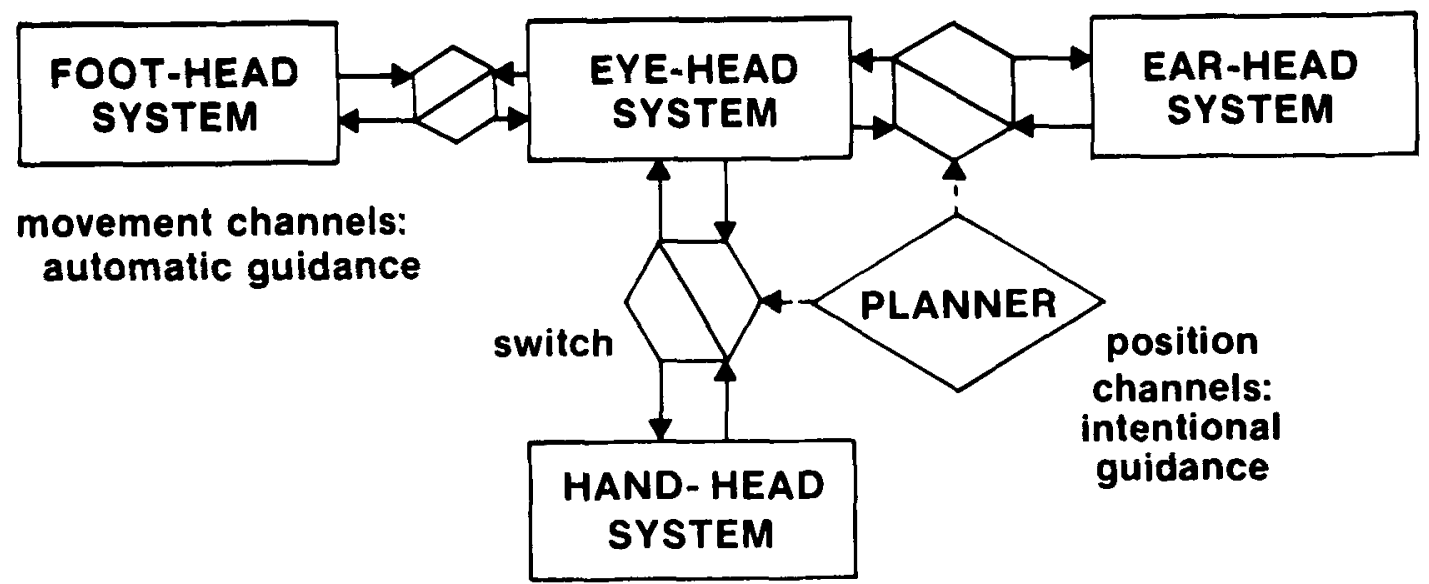

\begin{abstract}
Figure 1. Directionality-of-guidance model for hall exposure. Locomotion is automatically guided by undistorted optical flow, whereas intentional guidance of locational responses based on distorted position is subject to interference from a secondary cognitive task, with consequentially less adaptation. The manner in which the directional linkages are shown would produce adaptation in the eye-head (visual) system. (From "Attention and Prism Adapation" by G. M. Redding, S. E. Clark, \& B. Wallace, 1985, Cognitive Psychology, 17, 1-25. Copyright 1985 by Academic Press. Reprinted by permission.)
\end{abstract}

matic manner. Positional information, however, is distorted by the prisms; coordinative linkages along position channels produce discordance between subsystems and require intentional guidance to perform the task. Such discordance prompts local recalibration (i.e., adaptation) of positional encoding to reduce the discordance and reestablish spatial correspondence between subsystems. ${ }^{2}$ The locus of discordance registration and consequent adaptation depends upon the direction of coordinative linkages required by the exposure task. Adaptation occurs in the guided system. For example, when subjects direct their eyes to look at another person speaking or an obstacle they have just bumped into, comparison of discordant signals and adaptive recalibration occur in the responding visual system. ${ }^{3}$ On the other hand, when a subject anticipates a proprioceptive encounter (by arm or shoulder) with a previously seen obstacle, discordance registration and recalibration occur in a proprioceptive system (e.g., hand-head).

Thus this directionality-of-guidance model makes four predictions about the determinants of prism adaptation in hall exposure: (1) Total adaptation should equal the sum of local adaptation in subsystems. Adaptation is a local response to discordance registered in particular subsystems, and any task that links adaptive subsystems should show only the amount of adaptation that resides in the component systems. (2) Adaptation should suffer interference from cognitive tasks. Directional linkage of visual and nonvisual systems requires central processing capacity. When such capacity is not available because it has been allocated, for example, to mental arithmetic, intersystem coordination is weakened, discordance registration is less frequent, and adaptation of any kind should be reduced. (3) Locomotion is automatic and is not sufficient for adaptation to occur. Optical flow is undistorted and should produce no discordance or consequent adaptation, and, if locomotion does not require central processing capacity (i.e., is automatic), locomotion should not suffer interference from cognitive tasks. (4) The particular exposure task demands determine the locus or loci of adaptation. Task structures that require hand-to-eye or ear-to-eye linkage should produce visual adaptation, but when a reverse linkage (e.g., eye-to-hand) is required, adaptation should be localized in other systems (e.g., proprioceptive hand-head).

Conformity with the first prediction is well established in the prism adaptation literature (e.g., Hay \& Pick, 1966; Redding, 1978; Templeton, Howard, \& Wilkinson, 1974; Wallace, 1977; Welch, 1974; Wilkinson, 1971), and although there are exceptions to additivity (e.g., Welch, Choe, \& Heinrich, 1974), such exceptions can be explained without violating the basic assumption of a linear system (Redding \& Wallace, 1976, 1978; Wallace \& Redding, 1979). The second prediction has been validated many times and under a variety of conditions that seem to exclude alternative explanations (see Redding et al., 1985; Redding \& Wallace, 1985a). Support for the third prediction comes from the repeated observation that the primary task of walking does not suffer interference from a secondary mental arithmetic task (Redding et al., 1985; Redding \& Wallace, 1985a), and from the fact that salient optical flow can support exceedingly high walking speeds without producing any adaptation (Redding $\&$ Wallace, 1985b, Experiment 1). Redding and Wallace (1985b, Experiments 2 and 3 ) provided a test of the fourth prediction by manipulating the availability of auditory stimuli as a source for visual locational response. When the source of the auditory stimuli was visible to the subject and therefore available to guide the visual system (i.e., ear-to-eye linkage), adaptation was largely visual in nature, but when the source of auditory stimuli was not visible, adaptation was localized largely in the hand-head (proprioceptive) system, presumably because coordinative linkage of systems consisted largely of visual anticipation of proprioceptive encounters with walls (i.e., eye-to-hand linkage). This demonstrated ability to reverse the relative magnitude of 
visual and proprioceptive adaptation by varying the structure of the exposure environment is at least consistent with the directionality-of-guidance model.

The present experiment was designed to test an alternative explanation of the effects of the task structure manipulation performed by Redding and Wallace (1985b, Experiment 3 ). In this earlier experiment, all subjects received mental arithmetic problems given by the experimenter, who accompanied each subject as he/she walked in the hall; the primary manipulation was whether or not the speaking experimenter was visible to the subject. When the experimenter was not visible, she followed directly behind each subject as he/she walked. For the - experimenter-visible condition, she stood in the middle of and near the end of the short hall while subjects walked toward her. In both conditions subjects walked near the right-hand wall of the hall and wore base-left prisms, which produced a rightward displacement of the subjects' visual field. Consistent with the model, when the experimenter was visible and auditory guidance of visual response was possible, visual shift (VS) was greater than proprioceptive shift (PS), but the reverse was true when the experimenter was not visible. Moreover, the sum of VS + PS equaled the total shift (TS) measured for the linked eye-hand system in all conditions. An alternative account of these results involves presumed effects of changing head posture. The following argument is rather lengthy, because the logic of head-posture effects has not previously been fully explicated.

When subjects first put on prisms in hall exposure, the visual appearance of the world is approximately as it would be if the head were turned in the base direction by the amount of the displacement. That is, the hallway appears to angle off in the displacement direction, as it would if the head were turned in the base direction. An immediate effect is that the subject perceives the head to be turned in the base direction even though it remains straight on the shoulders. This discordance between actual head position and head position indicated by the angled view of the hall may form the basis for a more enduring perceptual recalibration, so that the head comes to feel turned in the base direction when it is straight on the shoulders and even without the discordance-producing visual input (i.e., an aftereffect). The predicted head shift (HS) is then in the direction of the displacement (i.e., when asked to position the head straight on the shoulders without vision, subjects should turn it in the displacement direction).

Note that if the head is kept straight during exposure, the eyes must turn in the displacement direction to fixate in the direction of locomotion (as specified by the optical flow pattern and hallway structure). Thus, eye position is asymmetrical and there is a basis for eye muscle potentiation aftereffects (e.g., Ebenholtz, 1976) to appear on a VS test that is referenced to the nose (i.e., when the subject sets a target to appear straight ahead of the nose).

However, the head is not usually kept perfectly straight. There is a (sometimes slight) tendency to "straighten" the head, turning it in the displacement direction (Kohler, 1964, p. 38). Such head turning is greatest when subjects are near the wall on the base side, because all they see without turning their heads is the near wall; in order to see any of the hall itself, they must turn their heads to a greater extent than when the nearest wall is on the displacement side. This head turning tends to center the structured array and direction of locomotion in the available visual field, thereby better enabling locomotion. Now, if a subject feels his/her head to be straight when it is turned in the displacement direction (as he/she should, since the immediate effect is to feel the straight head turned in the base direction), the difference between felt and actual head position may form the basis for a more enduring change in felt head position, so that the head comes to feel straight on the shoulders when it is turned in the displacement direction even without the visual input (i.e., an aftereffect). Again the predicted HS is in the direction of the displacement. Note, however, that this need not be a true perceptual recalibration effect (which is presumed to be based directly on discordant sensory inputs), but may rather be a purely local change based solely on a departure from the usual perceptual significance associated with potentiation of the neck muscle group (e.g., Ebenholtz, 1976) or with input from joint receptors in the neck (Howard \& Anstis, 1974). ${ }^{4}$

Again, note that to the extent that the head is turned in the displacement direction, the eyes will be symmetrically positioned in the head to fixate the center of the structured array and the direction of locomotion. Thus, head turning in the direction of displacement reduces any eyemuscle potentiation component of VS. On the other hand, such head turning should increase postural aftereffects in the neck and should increase this component of a PS test that is referenced to the head (e.g., in which the subject points straight ahead of the nose without vision).

Redding and Wallace (1985b) used rightward displacement, which would have tended to produce rightward head turning. Such head turning may have been slight because subjects always walked nearer the right wall, and only small head turns were necessary to avoid the "looming" near wall and to see the hall. However, even slight head turns may have large consequences. Solely from an eye and head posture viewpoint, the expected results of rightward displacement and a near right wall would normally be small amounts of VS (because rightward head postures would tend to center the eyes in the head, and therefore subjects would set a target straight ahead of their noses) and large amounts of PS (because the straight head would feel turned left and therefore subjects would point more left). This, of course, was the obtained result when the experimenter was not visible. When the experimenter was visible, however, she was always to the subjects' left. In order to see the experimenter, subjects might have tended to turn their heads left, thereby reducing asymmetrical head posture (and PS) while increasing asymmetrical eye posture (and VS). Thus, contrary to Redding and Wallace $(1985 \mathrm{~b}$, Note 5$)$, there does seem to be a clear 
postural basis for expecting the obtained changes of opposite direction in VS and PS.

To test this hypothesis, in the present experiment we repeated Redding and Wallace's (1985b) study with the addition of leftward, as well as rightward, displacement. If the original results were largely or entirely due to differences in eye and head posture, then one would expect the interaction to entirely disappear or even to suffer a reversal with leftward displacement. When the experimenter is not visible the presence of the near wall on the right (base) side should induce leftward head turns and symmetrical eye postures, with consequent large HS (appearing on the PS test) and smaller VS. This VS-PS difference should be larger than with rightward displacement. The effect of the visible experimenter should be to increase the magnitude of leftward head turning, increasing HS (and PS) still more and further reducing VS, perhaps even producing negative VS if head turning were great enough to produce asymmetrical eye posture opposite the direction of the displacement. On the other hand, if postural aftereffects were not largely responsible for the original interaction of adaptive locus and experimenter visibility, a similar pattern of results should appear for both displacement directions, although for leftward displacement the magnitude of the interaction might be reduced if there are any postural aftereffects in this situation.

\section{METHOD}

\section{Subjects}

The 48 subjects were right-handed undergraduate volunteers at Cleveland State University. All had normal or corrected-to-normal (contact lenses only) vision.

\section{Exposure Conditions}

All subjects walked back and forth along a short hall $(9.1 \mathrm{~m}$ in length $\times 1.7 \mathrm{~m}$ in width) for $10 \mathrm{~min}$. They wore goggles that afforded a monocular (right eye) view through a Risley prism set to laterally displace the visual field $30 \mathrm{D}\left(17.1^{\circ}\right)$ to the right or left. Subjects walked a path down the right side of the hall, made a U-turn near the end, and walked back down the other side. Pieces of tape (not visible to the subject) placed on the floor facilitated the recording of walking rates. Four groups of 12 subjects each received different treatments determined by the factorial combinations of rightward or leftward displacement and experimenter visible or not.

All groups received arithmetic problems created by generating all the unordered pairs of the double-digit numbers from 70 to 99 , excluding identical pairs. These 435 problems were randomly ordered prior to the experiment and were presented aloud to subjects in the form of addition questions (e.g., " $95+79=$ ?'”). Subjects were required to solve as many problems as possible, as accurately and as quickly as possible, while walking in the hall, and the accompanying experimenter recorded the number of problems attempted and the number correctly solved.

For the two conditions in which the experimenter was visible to the subjects, he stood in the middle and toward the end of the hall, with the subject making his/her turn behind the position of the experimenter. While a subject was making a turn and the experimenter was not visible, the experimenter moved quickly to the opposite end of the hall and was present in the subject's field of view when the turn was completed. For the two conditions in which the ex- perimenter was not visible, he followed a path directly behind the subject and never appeared in the subject's visual field.

The hall was the same one used in previous studies (Redding \& Wallace, 1985a, 1985b), and there was little human traffic except for the experimenter and subject. However, a restroom located off the hall was the occasion for infrequent pedestrian incursions from other parts of the building, and maintenance personnel also occasionally appeared in the hall. Although these interruptions were not usually accompanied by an exchange of speech, there were associated sounds of footsteps and doors, but the sources of these sounds were infrequently visible to the subjects. The only other detectable source of auditory stimuli was an elevator, not visible to the subjects. The hall was free of any obstacles and was interrupted only by the facings of closed doors. As in previous experiments employing arithmetic problems (Redding et al., 1985; Redding \& Wallace, 1985a, 1985b), the subjects were encouraged to walk rapidly and were instructed not to look at their hands or feet.

\section{Tests}

Before and after the 10-min hall exposure, subjects were tested 10 times on each of three tests with the prism set to $0 \mathrm{D}$. The test for VS required the subject to indicate verbally when a vertical line $(8 \mathrm{~cm}$ long $\times .2 \mathrm{~cm}$ wide and centered vertically in the subject's visual field at a viewing distance of $60 \mathrm{~cm}$ ) appeared to be straight ahead of his/her nose when moved laterally across the visual field by the experimenter. Starting position of the test line was random but equally frequent in left and right visual fields. The test for PS required the subject to point straight ahead of his/her nose with the right hand, without vision. A TS measure of both VS and PS was obtained by having the subject point at the objectively straight-ahead test line with his/her unseen right hand. For both VS and TS tests, the visible field was illuminated but homogeneous except for the target line. All 10 measures of one test were obtained before another test was given, but the order of tests was otherwise random across subjects. Level of adaptation was measured by the difference in arc degrees between average pretest and posttest performance, the adaptive direction of change being in the direction of the displacement for the VS test and opposite the displacement for the PS and TS tests. (For additional details of test procedures and apparatus, see Wallace \& Redding, 1979.)

\section{RESULTS}

Data for each of the three adaptation measures are displayed in Table 1 . The only significant source of variance in an analysis of the VS and PS data was the twoway interaction of experimenter visibility and locus of adaptation $[F(1,44)=16.09, p<.001]$. Clearly, VS was greater when the experimenter was visible but PS was greater when the experimenter was not visible, and this pattern was the same for both displacement directions $[F(1,44)<1.00]$. Comparison of the sum of VS+PS and TS revealed no significant sources of variance. All $F$ ratios were less than 1.0. Again clearly, VS +PS (4.0) is not different from TS (4.1), and additivity is virtually perfect in these data.

Subjects walked faster $[F(1,44)=10.80, p<.01]$ when the experimenter was visible $(65.3 \mathrm{~m} / \mathrm{min})$ than when he was not visible $(61.1 \mathrm{~m} / \mathrm{min})$, and the percentage of correctly solved problems was greater $[F(1,44)=$ $38.50, p<.001]$ when the experimenter was visible (85) than when he was not visible (77). However, the total 
Table 1

Mean Level of Adaptation (in Degrees) and 95\% Confidence Limits (in Parentheses) for Measures of Visual Shif (VS), Proprioceptive Shift (PS), and Total Eye-to-Hand Shift (TS)

\begin{tabular}{cccc}
\hline $\begin{array}{c}\text { Experimenter } \\
\text { Visible }\end{array}$ & \multicolumn{1}{c}{ VS } & PS & TS \\
\hline & \multicolumn{3}{c}{ Rightward Displacement } \\
Yes & 2.6 & 1.5 & 4.2 \\
& $(.7)$ & $(.4)$ & $(.9)$ \\
No & 1.4 & 2.5 & 4.1 \\
& $(.7)$ & $(1.1)$ & $(1.2)$ \\
Mean & 2.0 & 2.0 & 4.1 \\
& $(.5)$ & $(.6)$ & $(.7)$ \\
& Leftward Displacement & \\
Yes & 2.4 & 1.4 & 4.0 \\
& $(.8)$ & $(.5)$ & $(1.1)$ \\
No & 1.4 & 2.6 & 3.9 \\
& $(.8)$ & $(.6)$ & $(.6)$ \\
Mean & 1.9 & 2.0 & 4.0 \\
& $(.5)$ & $(.6)$ & $(.6)$ \\
Mean & 2.0 & 2.0 & 4.1 \\
& $(.4)$ & $(.4)$ & $(.4)$ \\
\hline
\end{tabular}

number of problems attempted was not different whether the experimenter was visible (58.2) or not (59.2), and there were no other significant sources of variance in the walking rate or problem performance data.

\section{CONCLUSIONS}

There is no evidence of postural effects in these data. Certainly, the relative magnitude of VS and PS did not vary as predicted from head and eye postures with leftward displacement. ${ }^{5}$ The data for both displacement directions very closely replicate previous results with rightward displacement (Redding \& Wallace, 1985b), and thus support the directionality-of-guidance model of prism adaptation in hall exposure. In this situation, a visible sound source provides the opportunity for auditorially guided visual locational responses, with consequent registration of discordance and perceptual recalibration in the visual (eye-head) system. The effects of experimenter visibility on walking rate and problem performance found in the present experiment did not occur in previous experiments, and may reflect greater motivational value of the visible experimenter for these tasks. In any case, these effects are not reflected in adaptation measures and do not offer competing explanations.

Finally, it should be reiterated that it is the role of the speaking experimenter as a guiding source for a visual locational response, not the mere fact that the sound source is visible, that is assumed to be the important determinant of visual adaptation. For discordance registration and perceptual recalibration to occur in the visual system, the speaking experimenter must act as an auditory stimulus guiding the subjects' looking behavior. Subjects must listen and then look for visual adaptation to occur. In the present situation the speaking experimenter often appeared at the edge or was even outside the restricted field of view, and looking for him frequently involved use of the audi- tory information. In other situations subjects might first look and then listen; thus the guidance linkage would be reversed, and the locus of adaptation should be in the auditory system.

\section{REFERENCES}

Craske, B., \& Crawshaw, M. (1974). Adaptive changes of opposite sign in the oculomotor systems of the two eyes. Quarterly Journal of Experimental Psychology, 26, 106-113.

Craske, B., \& Crawshaw, M. (1978). Spatial discordance is a sufficient condition for oculomotor adaptation to prisms: Eye muscle potentiation need not be a factor. Perception \& Psychophysics, 23, 75-79.

Ebenholtz, S. M. (1974). The possible role of eye-muscle potentiation in several forms of prism adaptation. Perception, 3, 477-485.

EBENholtZ, S. M. (1976). Additivity of aftereffects of maintained head and eye rotations: An alternative to recalibration. Perception \& Psychophysics, 19, 113-116.

Fitch, H. L., Tuller, B., \& Turvey, M. T. (1982). The Bernstein perspective: III. Tuning of coordinative structures with special reference to perception. In J. A. S. Kelso (Ed.), Human motor behavior. Hillsdale, NJ: Erlbaum.

HARRIs, C. S. (1980). Insight or out of sight?: Two examples of perceptual plasticity in the human adult. In C. S. Harris (Ed.), Visual coding and adaptability. Hillsdale, NJ: Erlbaum.

HAY, J. C., \& PICK, H. L., JR. (1966). Visual and proprioceptive adaptation to optical displacement of the visual stimulus. Journal of Experimental Psychology, 71, 150-158.

Howard, I. P., ANSTIS, T. (1974). Muscular and joint-receptor components in postural persistence. Joumal of Experimental Psychology, 103, 167-170.

KoHLER, 1. (1964). The formation and transformation of the perceptual world. Psychological Issues, 3(4), 1-173.

LeE, D. N., \& ThOmSon, J. A. (1982). Vision in action: The control of locomotion. In D. J. Ingle, M. A. Goodale, \& R. J. W. Mansfield (Eds.), Analysis of visual behavior. Cambridge, MA: MIT Press.

Longuet-Higgins, H. C., \& Prazdny, K. (1980). The interpretation of a moving retinal image. Proceedings of the Royal Society of London, Series B, 208, 385-397.

PosNer, M. I., \& SNYDER, C. R. R. (1975). Attention and cognitive control. In R. L. Solso (Ed.), Information processing and cognition. Hillsdale, NJ: Erlbaum.

Redding, G. M. (1978). Additivity in adaptation to optical tilt. Journal of Experimental Psychology: Human Perception \& Performance, 4, 178-190.

Redding, G. M., Clark, S. E., W Whlace, B. (1985). Attention and prism adaptation. Cognitive Psychology, 17, 1-25.

Redding, G. M., \& Wallace, B. (1976). Components of displacement adaptation in acquisition and decay as a function of hand and hall exposure. Perception \& Psychophysics, 20, 453-459.

Redding, G. M., \& Wallace, B. (1978). Sources of "overadditivity" in prism adaptation. Perception \& Psychophysics, 24, 58-62.

Redding, G. M., \& Wallace, B. (1985a). Cognitive interference in prism adaptation. Perception \& Psychophysics, 37, 225-230.

Redding, G. M., Wallace, B. (1985b). Perceptual-motor coordination and adaptation during locomotion: Determinants of prism adaptation in hall exposure. Perception \& Psychophysics, 38, 320-330.

SCHNEIDER, W., \& SHIFFrIN, R. M. (1977). Automatic and controlled information processing in vision. In D. LaBerge \& S. J. Samuels (Eds.), Basic processes in reading. Hillsdale, NJ: Erlbaum.

TEMPleton, W. B., Howard, I. P., Wilkinson, D. A. (1974). Additivity of components of prismatic adaptation. Perception \& Psychophysics, 15, 249-257.

THOMSON, J. A. (1983). Is continuous visual monitoring necessary in visually guided locomotion? Joumal of Experimental Psychology: Human Perception \& Performance, 9, 427-443.

WALLACE, B. (1977). Stability of Wilkinson's linear model of prism adaptation over time for various targets. Perception, 6, 145-151.

Wallace, B., \& Redding, G. M. (1979). Additivity in prism adaptation as manifested in intermanual and interocular transfer, Perception \& Psychophysics, 25, 133-136. 
WELCH, R. B. (1974). Speculations on a model of prism adaptation. Perception, 3, 451-460.

Welch, R. B., Choe, C. S., \& Heinrich, D. R. (1974). Evidence for a three-component model of prism adaptation. Joumal of Experimental Psychology, 103, 700-705.

WiLKINSON, D. A. (1971). Visual-motor control loop: A linear system? Journal of Experimental Psychology, 89, 250-257.

\section{NOTES}

1. The term automatic is operationally defined as the absence of interference from imposition of a secondary cognitive load (e.g., Posner \& Snyder, 1975; Schneider \& Shiffrin, 1977).

2. The reader should refer to Redding and Wallace (1985b) for an explication of the internal adaptive mechanism assumed to reside in each subsystem. Specifically, it should be noted that although discordance occurs between positional signals from the visual (eye-head) system and positional signals from other subsystems (e.g., the hand-head, proprioceptive system), the actual comparison of discordant positional signals and consequent perceptual recalibration is assumed to occur in the guided system.

3. The term visual adaptation is used to designate adaptive change in the eye-head system, which has phenomenal consequences for visual perception. The basic nature of such adaptation may be a change either in retinal local sign or in registered eye position (e.g., Craske \& Crawshaw, 1974; Harris, 1980). Current theory does not permit a test between these two possible accounts of visual change, and the present research is neutral with respect to this problem. Proprioceptive adaptation refers to any change in felt position of body parts. In the present study, the tests are sensitive to any such proprioceptive shift between the hand and head.
4. The term perceptual recalibration is reserved for calibration processes that determine the deviation of the guided system from the (external) standard provided by the guiding system so as to ascertain the proper correction factors. Muscle potentiation effects, as described by Ebenholtz (1974), or joint-receptor "adaptations," as described by Howard and Anstis (1974), do not involve any reference to an external standard, but, more simply, arise from a shift in resting position for a muscle group or joint receptor produced by atypical exercise. Both perceptual recalibration and postural aftereffects have perceptual consequences, and both are assumed to arise from mechanisms located within a subsystem, but the underlying mechanisms are different in nature. Recalibration requires input from an external discordant system, whereas postural aftereffects do not.

5. It may be noted that direct measures of head shift are not critical for present purposes. The present question was not whether head shift occurs. Very possibly, perceptual recalibration of head position does happen, and it may appear as part of the present PS measures (although a subject's encountering walls with his/her shoulder and arm also provides a possible basis for change in felt arm position). The present question was whether combination of head and eye posture and associated postural aftereffects could explain the reversal in relative magnitude of VS and PS. Measures of head shift are not directly relevant, because any change in felt head or eye position could be explained either as postural aftereffect or as perceptual recalibration. Any test between these hypotheses requires the kind of exposure manipulation performed in the present experiment, which may be expected to differentially affect the two mechanisms (cf. Craske \& Crawshaw, 1978). Direct measures of head shift would be useful, but they are not critical for purposes of the present investigation.

(Manuscript received August 22, 1986; revision accepted for publication March 2, 1987.) 